\title{
Síndrome de Wallenberg secundária a aneurisma gigante de artéria vertebral
}

\author{
Rodrigo Antônio Rocha da Cruz Adry,2, Catarina Couras Lins ${ }^{3}$, \\ Marcio Cesar de Mello Brandão ${ }^{4}$ \\ Hospital Geral Roberto Santos e Hospital Geral do Estado da Bahia.
}

\begin{abstract}
RESUMO
Descrevemos um caso de síndrome bulbar lateral, também conhecida pelo epônimo síndrome de Wallenberg, em uma paciente do sexo feminino de 46 anos, com todos os sinais e sintomas clássicos, determinada por aneurisma gigante do segmento intracraniano da artéria vertebral. A síndrome de Wallenberg, apesar de comum a sua apresentação como consequência de aneurisma gigante de artéria vertebral, não é frequente, e na patogênese da sintomatologia o efeito de massa dessas lesões deve ser considerado.
\end{abstract}

PALAVRAS-CHAVE

Síndrome de Wallenberg. Aneurisma intracraniano gigante.

\begin{abstract}
\section{KEYWORDS}

Wallenberg's syndrome. Giant intracranial aneurysm.
\end{abstract}

Wallenberg's syndrome due to giant aneurysm of the vertebral artery

We present a case of lateral bulbar syndrome, or Wallenberg's syndrome, in a 46 year-old woman with all classic signs and symptoms, due to giant aneurysm of the vertebral artery at its intracranial segment. Even though common, Wallenberg syndrome due to giant aneurysm is not frequent, and in its pathogenesis the mass effect of this lesions may have a significant role.

\section{Introdução}

A síndrome de Wallenberg pode ser caracterizada como parte das síndromes isquêmicas vertebrobasilares laterais. ${ }^{9}$ Ela é sinônimo da síndrome bulbar lateral e constitui o protótipo das síndromes que afetam os núcleos dos pares cranianos IX e X..$^{5}$ É a síndrome mais comum que acomete a medula oblonga. ${ }^{8}$ É a apresentação clínica mais comumente encontrada nos casos de aneurismas dissecantes da artéria vertebral em seu segmento intracraniano; esses aneurismas são pouco comuns, tendo como principal consequência o acidente vascular cerebral da circulação posterior com isquemias no tronco cerebral e hemisférios cerebelares, com incidência que varia entre $26 \%$ e $43 \% .^{1}$ Essa síndrome resulta do infarto do bulbo pela trombose da artéria vertebral ou pela dissecção desta que condicione a oclusão da abertura da artéria cerebelosa posterior inferior (ACPI), com predisposição especial por grupos de pacientes da terceira idade. ${ }^{3}$
São definidos como gigantes os aneurismas intracranianos com diâmetro superior a $25 \mathrm{~mm}$, sendo sua incidência estimada em $2 \%$ a $5 \%$ de todos os aneurismas. Apesar de poderem ser encontrados em qualquer lugar da árvore arterial cerebral, os aneurismas gigantes seguem uma prevalência topográfica um tanto diferente da dos aneurismas não gigantes: $34 \%$ a $67 \%$ na artéria carótida interna, $11 \%$ a $40 \%$ nas artérias cerebrais anterior e média e $13 \%$ a $56 \%$ na artéria basilar ou vertebral. ${ }^{4}$

\section{Relato do caso}

Paciente de 46 anos, do sexo feminino, negra, natural e procedente de Salvador/BA, procurou o ambulatório de neurologia, uma semana após receber alta de serviço de emergência público da cidade, onde deu entrada com quadro de vertigem, vômitos, disartria,

1 Médico-residente em Neurocirurgia do Hospital de Base de São José do Rio Preto - Faculdade de Medicina de São José do Rio Preto (Famerp). 2 Médico do Serviço de Neurologia e Neurocirurgia do Hospital Geral do Estado da Bahia e Hospital Geral Roberto Santos.

3 Acadêmica de Medicina da Escola Bahiana de Medicina e Saúde Pública (Bahiana).

4 Chefe do Serviço de Neurologia e Neurocirurgia do Hospital Geral do Estado da Bahia e Hospital Geral Roberto Santos. 
parestesia no hemicorpo direito e tendência à queda para esquerda quando em postura ortostática, que foi avaliado pela própria paciente e por equipe assistente, segundo ela, como déficit de força em hemicorpo esquerdo. Permaneceu internada por 96 horas, com melhora parcial dos sintomas, sendo encaminhada para ambulatório. Saiu com diagnóstico de acidente vascular cerebral, sem saber especificar maiores detalhes e sem relatório de alta, mas tendo consigo tomografia axial computadorizada não contrastada de crânio sem alterações patológicas.

Como antecedentes mórbidos pregressos, ressalta-se hipertensão arterial sistêmica (HAS), em uso há quatro anos de captopril $25 \mathrm{mg}$ de 12/12h, com níveis tensionais prévios em torno de 140 x $90 \mathrm{mmHg}$. História familiar de HAS (mãe, duas tias maternas e um irmão) e acidente vascular encefálico (mãe, falecida há dois anos). Nega tabagismo e etilismo.

Apresentava-se na primeira avaliação ambulatorial, 12 dias após o início dos sintomas, sem alterações no exame físico geral. Ao exame neurológico, estava vigil, com funções mentais superiores preservadas, nuca livre, miose, ptose palpebral incompleta, enolftalmia e anidrose à direita, nistagmo horizontal com componente rápido para direita, paresia do palato mole à direita; sinal da cortina à direita, disfonia, rouquidão, força muscular preservada bilateralmente, hipoestesia superficial térmico-dolorosa à direita na face e contralateral no tronco e membros superior e inferior, dismetria e disdiadococinesia à direita, distasia e disbasia com lateropulsão à direita, reflexos miotáticos ausentes proximais e presentes $(+/++++)$ distais em membros inferiores (aquiliano e patelar), simétricos, sem sinais de libertação piramidal. Os achados ao exame físico podem ser então resumidos em: síndrome de Claude Bernard-Horner (miose, ptose palpebral incompleta, enolftalmia e anidrose) à direita; síndrome vestibular (nistagmo, vertigem, lateropulsão); hemiataxia tipo cerebelar (dismetria, disdiadococinesia, à direita; distasia e disbasia); paresia do glossofaríngeo/vago à direita (paresia do palato mole à direita; sinal da cortina à direita, disfonia, rouquidão) e hipoestesia superficial térmico-dolorosa cruzada.

Tais achados sindrômicos compõem a plêiade da síndrome de Wallenberg, indicando como topografia lesional a região dorsolateral do bulbo. A ressonância nuclear magnética do encéfalo evidenciou lesão sacular em topografia de artéria vertebral direita em contato com a porção retro-olivar do bulbo, compatível com aneurisma gigante desse vaso (Figuras 1 e 2).

Paciente em programação para estudo angiográfico da circulação cerebral e posterior abordagem eletiva neurocirúrgica, de acordo com a disponibilidade do serviço de neurocirurgia.

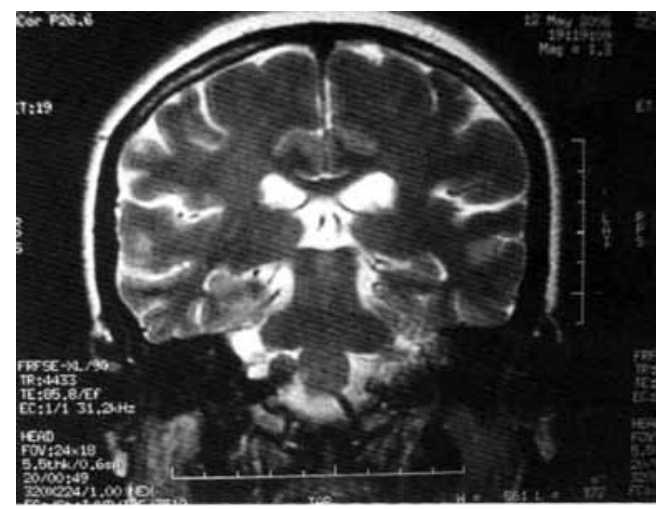

Figura 1 - Aneurisma gigante sacular da artéria vertebral direita na altura da origem da artéria cerebelar posterior inferior. Visão coronal na ressonância nuclear magnética do encéfalo, em T2.

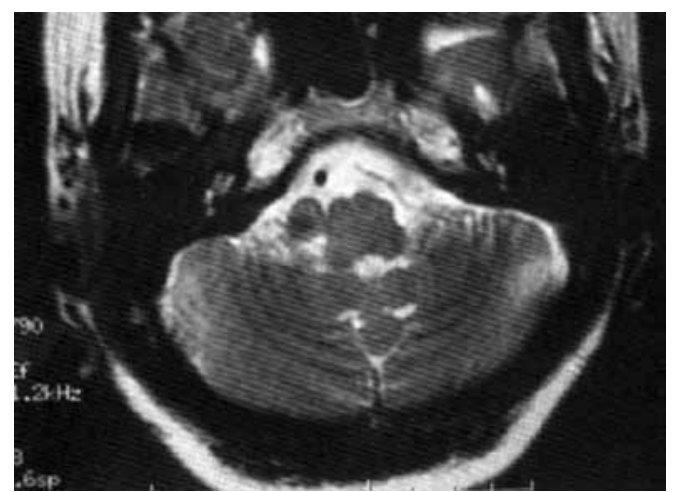

Figura 2-Aneurisma gigante sacular da artéria vertebral direita na altura da origem da artéria cerebelar posterior inferior. Visão axial na ressonância nuclear magnética do encéfalo, em T2.

\section{Discussão}

A síndrome de Wallenberg pode se apresentar com dor e parestesia facial ipsilateral, ataxia ipsilateral, vertigem, náuseas, vômitos e alterações na percepção da temperatura no lado contralateral da lesão. Outro achado nesses pacientes é a presença da síndrome de Horner ipsilateral (enoftalmia, ptose palpebral, miose e anidrose), devida ao envolvimento das fibras simpáticas descendentes do hipotálamo. ${ }^{3}$ A tríade síndrome de Horner, ataxia do membro ipsilateral e parestesia no membro contralateral pode indicar a presença de infarto bulbar. ${ }^{11}$ Todos os sinais e sintomas podem sugerir a síndrome de Wallenberg, mas sua confirmação deve ser feita por meio de exames de imagem. ${ }^{3}$ A síndrome de Wallenberg é a apresentação clínica mais comumente encontrada nos casos de aneurisma de artéria vertebral, cuja presença no segmento intracraniano dessa artéria é pouco comum, tendo como principal consequência o acidente vascular cerebral da circulação posterior com isquemias do tronco cerebral e hemisférios cerebelares, com incidência que varia entre $26 \%$ e $43 \%{ }^{5}$ 
A ressonância magnética encefálica é o padrão-ouro para o diagnóstico em virtude de sua capacidade superior, em comparação com a tomografia computadorizada, para a detecção de lesões dessa região. ${ }^{6}$

O prognóstico para a recuperação de um infarto bulbar normalmente é bom, embora em alguns casos possa levar ao rebaixamento do nível de consciência e até mesmo à morte, se houver presença de edema cerebral ou síndromes de herniação.

Apesar de a síndrome de Wallenberg ser uma apresentação comum para as lesões isquêmicas, é rara nos casos de aneurisma gigante de artéria vertebral. A patogênese da sintomatologia deve-se à compressão da dilatação vascular adjacente, bem como aos escapes hemáticos levando a sequestro de fluxo arterial para a região retro-olivar do bulbo. ${ }^{3}$

Há dois tipos principais de aneurismas gigantes: os saculares e os fusiformes, sendo muitas vezes difícil a diferenciação meramente morfológica entre essas duas formas. Os primeiros são mais frequentes nas regiões de bifurcações vasculares e são geralmente decorrentes de estresse hemodinâmico. Os últimos, também chamados de dissecantes, são decorrentes de aterosclerose, arteriopatias congênitas ou dissecção traumática, Vários fatores estão envolvidos na gênese de ambas as formas, como idade, HAS, tabagismo etc. Com base no aspecto macroscópico e na localização (bifurcação entre a artéria vertebral direita e artéria cerebelosa posterior inferior direita), classificamos como aneurisma gigante sacular a lesão de nossa paciente.

A apresentação clínica mais frequente dos aneurismas gigantes é a hemorragia subaracnóidea (HSA) não é o caso de nossa paciente. É relatada pela literatura uma taxa de apresentação de $80 \%$ dos casos de aneurismas gigantes como HSA. ${ }^{4} \mathrm{O}$ risco de sangramento anual desse aneurismas é maior do que o dos aneurismas menores. Um estudo multicêntrico publicado em 1998 pelo International Study of Unruptured Intracranial Aneurysms Investigators (Unruptured intracranial aneurysms-risk of rupture and risks of surgical intervention) encontrou um risco de sangramento anual de $6 \%$ para os aneurismas gigantes; um estudo mais recente no Japão mostrou que $80 \%$ a $100 \%$ dos pacientes com aneurismas gigantes sofrem danos neurológicos ou evoluem a óbito em torno de cinco anos. ${ }^{4}$ Os pacientes com aneurismas menores geralmente apresentam sintomas com o rompimento do aneurisma, enquanto os pacientes com aneurisma gigante apresentam primeiramente o seu efeito de massa.

No caso em questão, os sintomas e sinais são decorrentes não de sangramento subaracnóideo, mas do efeito compressivo da estrutura vascular contra a porção retro-olivar do bulbo. Vários autores descrevem como fator agravante da topografia em fossa posterior dos aneurismas gigantes ${ }^{10} \mathrm{o}$ fato de ser uma cavidade sem espaços residuais e onde até os movimentos posturais da cabeça podem exacerbar ou desencadear sintomas compressivos, muitas vezes fatais. ${ }^{4} \mathrm{Um}$ ótimo tratamento de aneurismas cerebrais requer sua exclusão da circulação intracraniana. ${ }^{7}$

Em relação ao tratamento, a literatura cita tanto as opções terapêuticas endovasculares quanto a cirurgia convencional com clipagem. Os novos dispositivos desenvolvidos nos últimos anos convertem aneurismas intratáveis em aneurismas de baixo risco, principalmente com a utilização de agentes líquidos associados a balões e stents na terapia endovascular. ${ }^{2} \mathrm{O}$ prognóstico dos pacientes com aneurismas não tratados, especialmente na circulação posterior, é extremamente pobre, a maioria $(80 \%$ a $100 \%)$ fica inválido ou morre durante o curso de cinco anos de seguimento. ${ }^{6}$ Em discussão com o Serviço de Neurocirurgia do hospital, optamos pela clipagem, uma vez que, em se tratando de lesões saculares de circulação de fossa posterior, esse procedimento tem melhores evidências de efetividade e segurança em longo prazo.

\section{Referências}

1. Andrade GC, Oliveira JG, Dauar RFB, Nalli DR, Braga FM. Aneurisma dissecante de artéria vertebral intracraniana fenestrada submetido ao tratamento endovascular. Arq Neuropsiquiatr. 2005;63(2-A):352-6.

2. Blasco J, Macho JM, Burrel M. Endovascular treatment of a giant intracranial aneurysm with a stent-graft. J Vasc Interv Radiol. 2004;15:1145-9.

3. Elizondo GV, Kawachi JN. Síndrome de Wallenberg. Médica Sur. 2005;12:212-4.

4. Hayashi T, Hirayama N. Severe brainstem compression by an unruptured giant vertebral aneurysm: an autopsy case. Legal Medicine. 2007;9:322-5.

5. Hosoya T, Watanabe N, Yamaguchi K, Kubota H, Onodera Y. Intracranial vertebral artery dissection in Wallenberg syndrome. Am J Neuroradiol. 1994;15:1161-5.

6. Kim JS, Lee JH, Suh DC. Spectrum of lateral medullary syndrome: correlation between clinical findings and magnetic resonance imaging in 33 subjects. Stroke. 1994;25:1405-10.

7. Komotar RI, Mocco J, Lavine SD, Solomon RA, Ricardo I. Angiographically occult, progressively expanding, giant vertebral artery aneurysm. J Neurosurg. 2006;105:1068-71.

8. Krasnianski M, Müller T, Stock K, Zierz S. Between Wallenberg syndrome and hemimedullary lesion: CestanChenais and Babinski-Nageotte syndromes. J Neurol. 2006;33:1442-6.

9. Love B, Biller J. Neurovascular system. In: Goetz G, editor. Textbook of clinical neurology. Philadelphia: Saunders; 2003, p. 203.

10. Morita KI, Inenaga C, Ito Y, Fujii Y, Tanaka R. An autopsy case of giant aneurysm of vertebrobasilar artery treated by endovascular surgery. Acta Neurochir (Wien). 2003;145:723-5. 
11. Sacco RL, Freddo L, Bello JA. Wallenberg's lateral medullary syndrome. Clinical magnetic resonance imaging correlations. Arch Neurol. 1993;50:609-14.

\section{Endereço para correspondência}

Rodrigo Adry

Rua Professor Enjolras Vampré, 90, ap. 25, São Manoel

15091-290 - São José do Rio Preto, SP, Brasil

E-mail: rodrigoadry@hotmail.com 